\title{
Measurements-based Modeling of Burst Errors on Multiple Parallel Storage Channels
}

\author{
Maria Varsamou and Theodore Antonakopoulos \\ Department of Electrical and Computers Engineering \\ University of Patras, Rio-Patras, 26500, Greece \\ Email: mtvars@upatras.gr, antonako@upatras.gr
}

\begin{abstract}
This paper presents a measurement-based burst error model for storage devices that use multiple parallel channels when they are affected by external disturbances. The burst errors are modeled using a set of Markov processes and channel error measurements are exploited to specify the parameters of the Markov processes and to determine the correlation of errors in the various channels. The application of the proposed model to AFM-based probe storage devices is presented and numerical results for various cases of external noise sources are given.
\end{abstract}

\section{INTRODUCTION}

In recent years, due to the constantly increasing need for higher data rates and larger storage densities, the use of multiple, simultaneously accessed channels in storage systems has been investigated [1]. The general architecture of such a system with parallel channels is shown in Fig. 1. The original data block is partitioned into several subsets, which are accessed concurrently, each one over a different storage channel. When the read/write operation of a new data block is initiated, a preamble is used for synchronization purposes and then a number of data packets is accessed. Each data packet consists of a subset of a user sector along with codingrelated data. A characteristic example of such a parallel system is found on probe-based storage devices [2], where ultrahigh storage densities and high data rates can be achieved by using atomic force microscope (AFM) techniques to write and read back data in very thin polymer films with the parallel operation of $2 \mathrm{D}$ arrays with multiple tips.

Several coding schemes for parallel systems can be used. One scheme with both random and burst error correction capabilities, which is based on Reed-Solomon codes along with proper interleaving is shown in Fig. 1. The original message is partitioned into a number of datawords which are then encoded with an RS code, usually on an 8-bits symbol basis (byte). The encoded data are symbol-interleaved and then split into smaller blocks, each for a single channel [3]. If the channels are statistically independent, then a burst of errors in a single channel will be spread across multiple codewords and the decoder stands good chances of correcting it. However, if an external noise source is applied to the whole system, then it affects all channels concurrently and with the same statistical characteristics. In this case, depending on the number of channels and the duration of the noise effect, a great number of errors appear in all codewords, the errors are correlated and the error correction mechanism may fail.

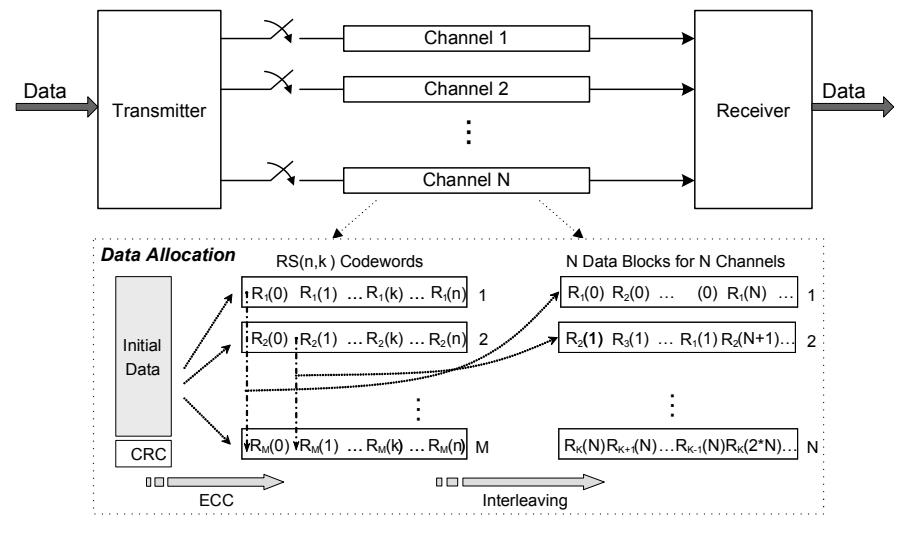

Fig. 1. The multiple storage channels system.

In order to study the relationship between the characteristics of the external noise source and the correlated channel errors that appear in the various channels, to derive the statistics of the symbol burst errors that affect the data codewords and to estimate their effects on the error correction capability of the coding scheme, an error model is needed. Such a model has to provide results for various system configurations and its parameters have to be based on a number of experimental data. In this work, we present a multiple-channels burst error model that employs Markov processes to describe the mechanism of burst errors that appear in a set of simultaneously accessed channels. Experimental measurements of the effect of external noise sources on the channels conditions are incorporated to control and configure the parameters of the Markov processes, thus making the model flexible for studying and evaluating the reliability of various coding schemes under various kinds of external disturbances. The proposed model is described in detail in Section II. The application of the proposed model on a storage device with multiple parallel probes, along with error distributions and statistics based on specific measurement scenarios, are presented in Section III.

\section{Measurement-Based Burst Error Channel MODEL}

The proposed burst error channel model, which is shown in Fig. 2, consists of two complementary modules. The first one is related to the modeling of the external noise source, while the second module models the correlated burst errors 


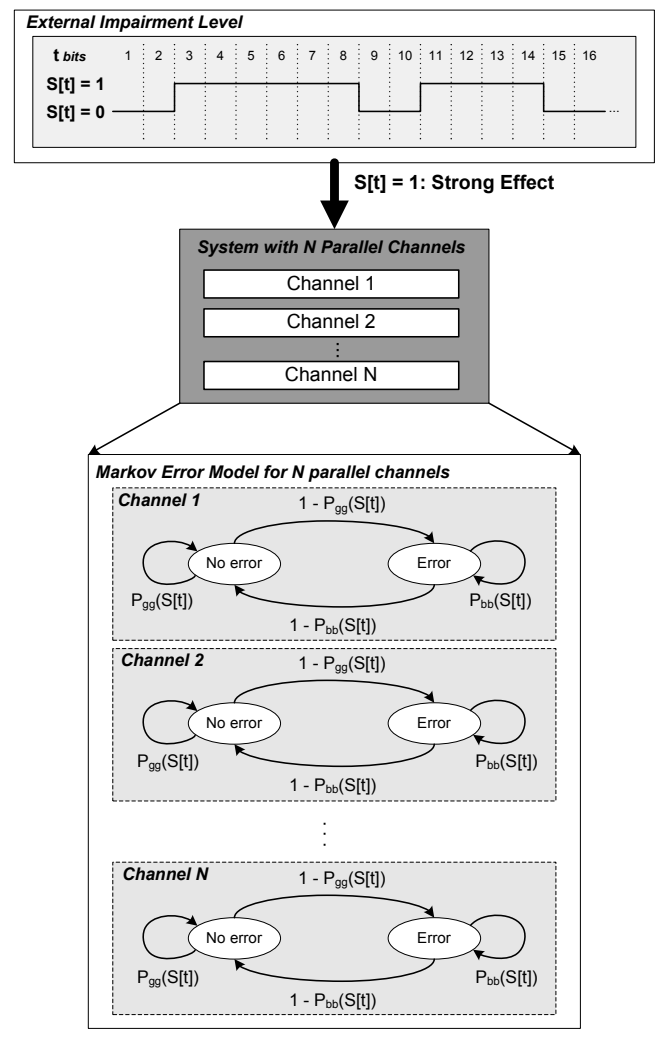

Fig. 2. The measurement-based burst error Markov model.

in the multiple storage channels. The bit error probability in a storage field depends on the statistical characteristics of the external disturbance.

Depending on the external noise characteristics, durations of high $(S[t]=1)$ and low $(S[t]=0)$ error probabilities are observed. In channels with line coding and intersymbol interference, error in detection of a bit affects the probability of error in the detection of adjacent bits. Depending on the characteristics of the external noise source and its effect on the error correction scheme, more that one levels of $S[t]$ may be needed to model the external impairment. Since the various storage channels are affected by the same noise source, a common Markov process models the error appearance in each separate channel. All $N$ channels enter the 'No error' or 'Error' state at the same bit offset from the synchronization preamble. When a channel is in the 'No error' state, an error occurs with probability $P=0$, whereas when it is in the 'Error' state, an error occurs with probability $P=1$. The transition probabilities from each state, $P_{g g}$ and $P_{g b}=1-P_{g g}$ for the 'No error' state, $P_{b b}$ and $P_{b g}=1-P_{b b}$ for the 'Error' state, depend on the statistical characteristics of the external noise source. This dependency on the external noise source expresses the spatial correlation among the errors in all channels. The sequence of these periods along with the state transition probabilities for each $S[t]$, are obtained by extensive measurements on an actual system.

The model is enhanced with statistical features, which enable the derivation of various probabilistic distributions, such as, the distribution of errors in the codewords and the number of codewords that cannot be decoded. Therefore, it forms a powerful tool for studying the reliability, in terms of error correction capability, of the coding scheme used in a storage system, when burst errors appear in all channels and evaluate the role of various parameters of the system, such as the number of channels and the interleaver depth.

\section{Modeling of Burst Errors in a Probe-based STORAGE DevicE}

An application of the aforementioned model has been applied in the probe-based data storage device presented in [1]. In this device, the information is stored by means of thermo-mechanical formation of indentations in thin polymer films [4], using nanometer-sharp tips, similar to those used in atomic force microscopy (AFM) [5], and ultra-high densities are achieved [6]. To increase the achievable data rates, the use of a large 2D array of probes has been proposed [7]. In this case, each probe performs read/write/erase operations on a dedicated area, named a data field, the storage medium is placed in the $x / y$ plane, and the relative motion is performed by using a highly accurate microscanner.

As in conventional storage devices, the data are stored in the form of sectors of fixed length. If $N$ is the number of probes operating in parallel, each sector is encoded as shown in Fig. 1, $N$ smaller blocks are formed, and each one is stored in a single storage field. During data reading, the microscanner moves the storage fields under their associated tips, such that each tip operates in the center of the line with the sequence of indentations corresponding to the specific sector. The read channel that corresponds to each probe, along with the effects of the various distortions of the readback signal has been studied in [8]. Since each probe operates on a distinct storage area, the $N$ parallel read channels are statistically independent, only when there is no external noise source. However, an external shock or vibration applied to the device, and consequently to the microscanner, while reading or writing a sector, will cause the same displacement to all tips. The effect of the microscanner perturbations on both $\mathrm{x}$ and $y$ directions on the positioning error and consequently on the probability of error in the bit sequence reproduced by the readback signal in a single channel, is studied in [9].

In the following sections, we show how the proposed model can be used to derive statistics and study the effect of various shock profiles on a probe-based storage device with certain design parameters. Additionally, we use measurements of external disturbances caused by accelerations imposed on the storage device, when it is attached on a running person and when it is placed on a desk, to configure the model and validate its effectiveness, by comparing it with experimental data provided by an accurate system simulator. 

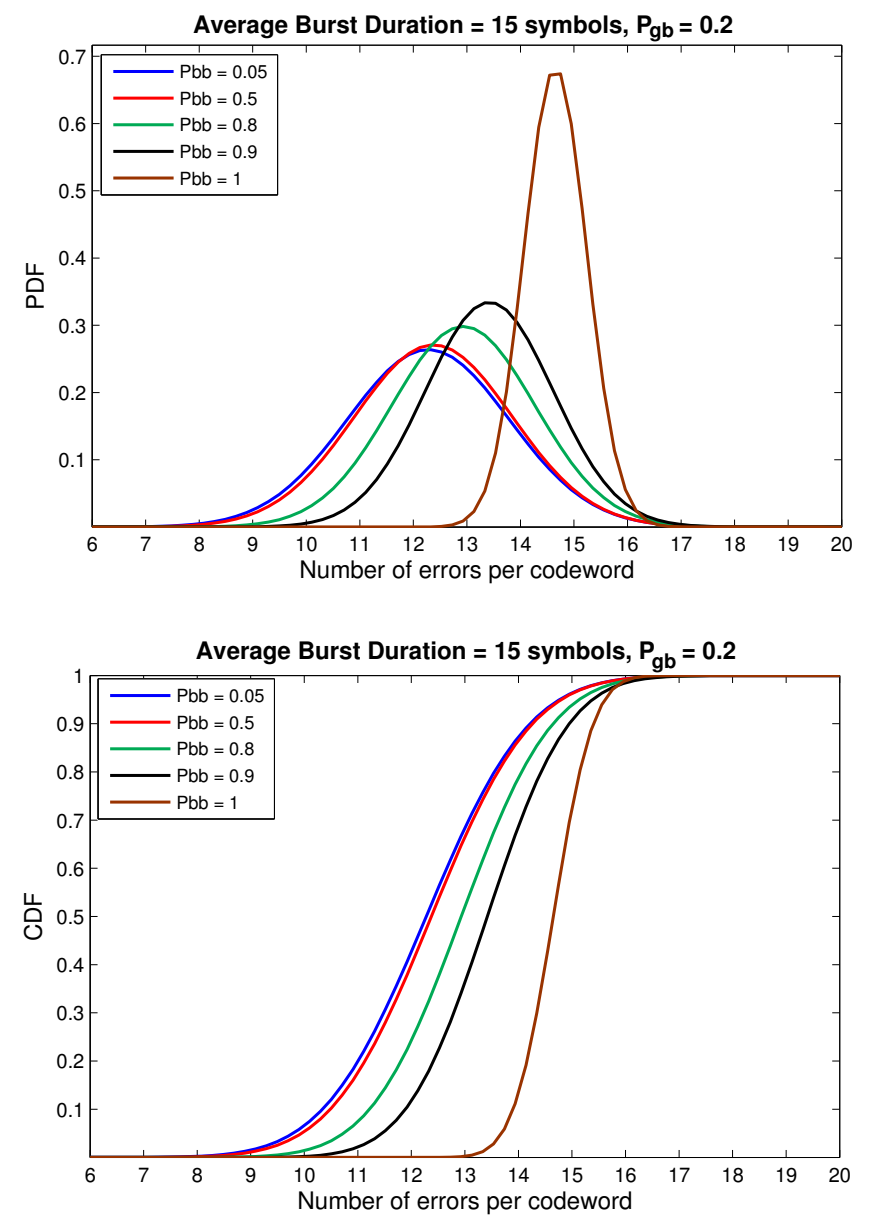

Fig. 3. Probability distributions of the number of errors per codeword when an external disturbance of average duration $B_{a v}=15$ symbols affects a system with $N=16$ fields and $M=16$ codewords, for $P_{g b}=0.2$ and several values of $P_{b b}$.

\section{A. Burst Error Model Simulation Results}

We use the proposed model to study the effect of various different realizations of an external disturbance with a standard duration of $15 \mathrm{RS}$ symbols that affects a storage device with 16 storage fields, a sector size of 2048 bytes and RS $(151,129)$ code, i.e. a sector is divided in 16 codewords. We assume that errors appear in the channels only when $S[t]=1$, which means that $P_{g b}(S[0])=0$. Different realizations of the disturbance give different values for $P_{g b}(S[1])=P_{g b}$ and $P_{b b}(S[1])=$ $P_{b b}$.

Fig. 3 and 4 show the probability distributions regarding the mean number of errors that appear in a codeword and the mean number of codewords that cannot be decoded for various scenarios, as they are derived from the statistics produced by the presented error model. Using mathematical fitting tools, these distributions are determined to be normal, with mean value and sigma that follow the curves shown in Fig. 5 and 6.

\section{B. Measurement-based Burst Error Model Parameterization}

In order to study the error correction capability of the coding scheme used in a probe-based storage device under
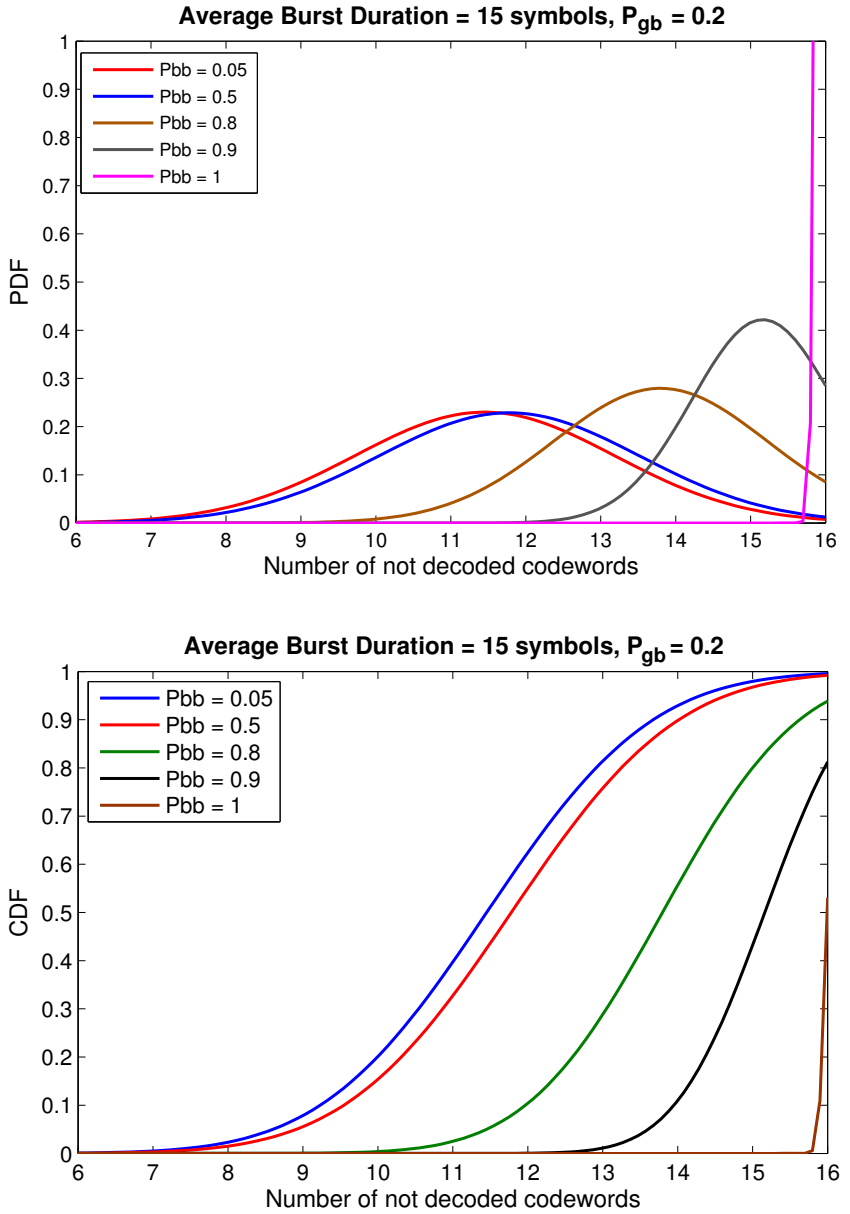

Fig. 4. Probability distributions of the number of not decoded codewords, when an external disturbance of average duration $B_{a v}=15$ symbols affects a system with $N=16$ fields and $M=16$ codewords, for $P_{g b}=0.2$ and several values of $P_{b b}$.

various physical disturbances, we need to configure the burst error model to match the effect of such disturbances. For that purpose, we use measurements produced by an accurate simulator of such a system. The simulator incorporates all system functionalities, i.e. the microscanner movement and the sensing capabilities, the readback signal of multiple data fields and the complete data mapping and coding scheme. It also includes the various noise sources that affect the readback signal, as they are studied in [8] and [9], thus producing realistic results. Furthermore, external disturbances in the form of acceleration measurements over time can be applied as an input to the system. Using this approach, the complete operation of reading and decoding the data written on multiple storage fields can be simulated. The detection and error decoding functionalities provide statistics regarding the bit errors that appear in each data field as well as the symbol errors that affect the RS codewords and the total number of codewords that cannot be decoded.

We use the simulator to get measurements and statistics that will be used for the estimation of the parameters of the burst error model, so that it can describe effectively the burst errors 

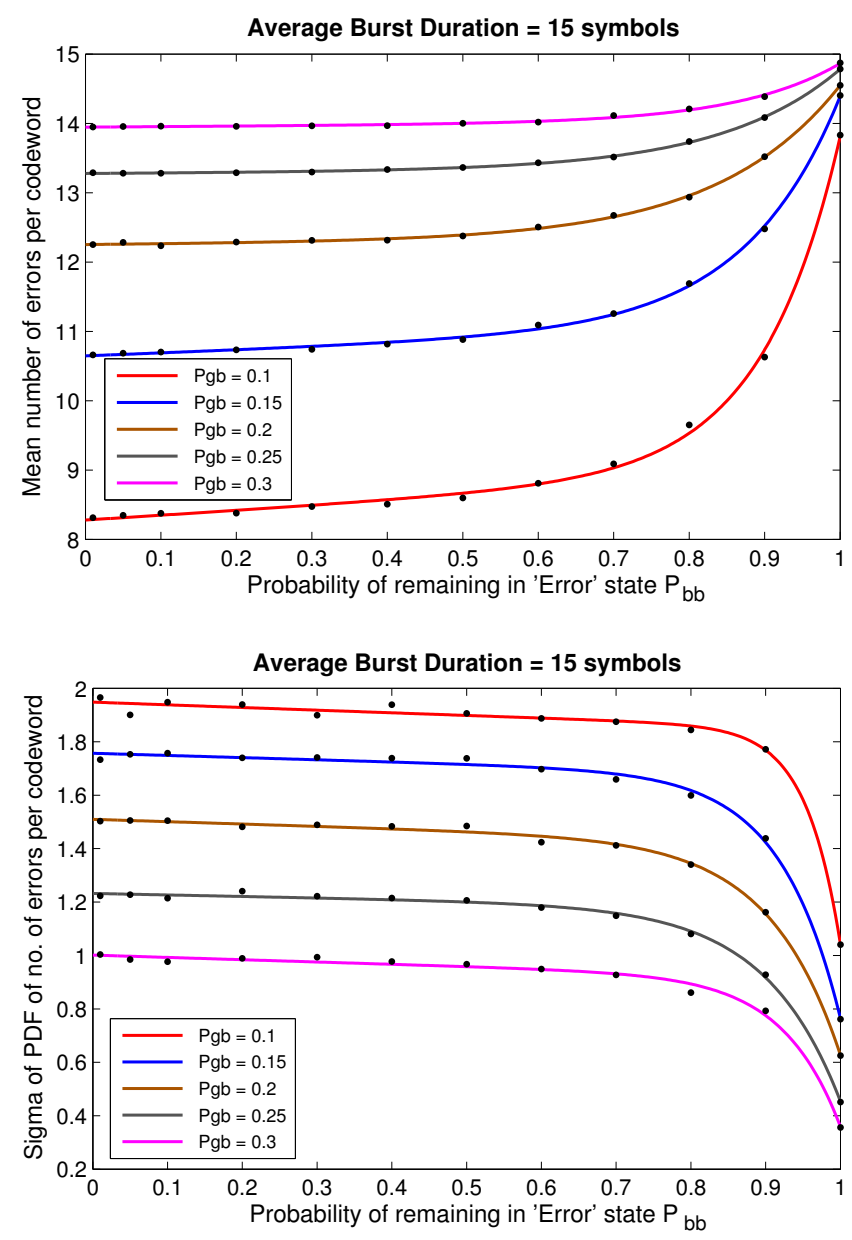

Fig. 5. Mean value and dispersion around mean versus $P_{b b}$ for the number of errors in a single codeword, when an external disturbance of average duration $B_{a v}=15$ symbols affects a system with $N=16$ fields and $M=16$ codewords, for various values of $P_{g b}$.

appearance, when the system is affected by actual physical disturbances caused by accelerations imposed on a storage device operating inside a mobile phone. Typical activities that may cause such disturbances are, for example, a running person and the placement of a mobile phone on a desk. An experimental setup for measuring the accelerations imposed on a mobile phone on all $\mathrm{x}, \mathrm{y}$ and $\mathrm{z}$ axis during various activities is presented in [10]. Using this setup, the respective disturbance profiles for the aforementioned activities were produced and given as input to the simulator.

For the measurements, we consider a system with $N=16$ data fields. The size of each data field is $100 u m$ in the $\mathrm{x}$ direction, the distance between adjacent lines is $40 \mathrm{~nm}$ in the $\mathrm{y}$ direction and the distance between adjacent symbols is $20 \mathrm{~nm}$ in the $\mathrm{x}$ direction. The linear velocity of the microscanner is $0.25 \mathrm{~nm} /$ us. During reading, 4 samples per symbol are used, while the system SNR is $12 d B$. Regarding the dataflow, a sector size of 2048 bytes is used and the data are encoded with an $R S(151,129)$ code. That means that $M=16$
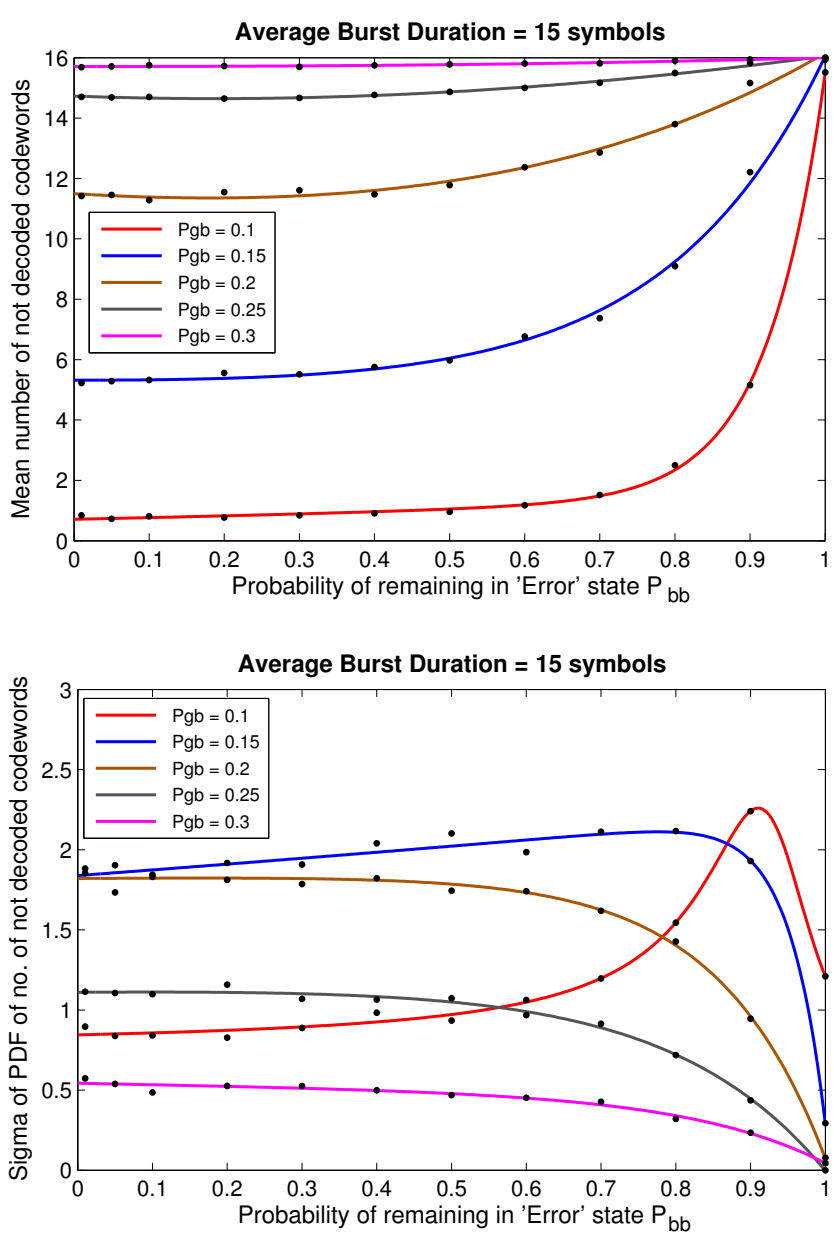

Fig. 6. Mean value and dispersion around mean versus $P_{b b}$ for the number not decoded codewords, when an external disturbance of average duration $B_{a v}=15$ symbols affects a system with $N=16$ fields and $M=16$ codewords, for various values of $P_{g b}$.

codewords are formed. The effect of the two different kinds of disturbances on the readback signal and the configuration of the respective burst error model are given below:

1) Disturbance caused on a storage device attached to a person running with a speed of $10 \mathrm{~km} / \mathrm{h}$ : The acceleration profile that affects the microscanner movement on both directions, produced by measurements on a mobile phone that uses the storage device and is attached on a person that runs at $10 \mathrm{~km} / \mathrm{h}$, is presented in Fig. 7(a). Note that this profile corresponds to a snapshot of the total periodic movement. The deviation of the tip from the center of the line along with its effect on the readback signal in one data field is shown in Fig. 7(b). It is obvious that during the disturbance, errors appear in the corresponding bits. The error locations in the affected bits from one data field are shown in Fig. 7(c). From the distribution of the bit errors, the External Impairment Level sequence that will be used to control the respective burst error model is derived. This sequence is depicted in Fig. 7(c). The errors from all data fields are also used to calculate the 
TABLE I

Markov Model Parameters AND COMPaRative Results With EXPERIMENTAL DATA FOR A SHOCK PROFILE CAUSED BY A PERSON RUNNING AT $10 \mathrm{KM} / \mathrm{H}$

\begin{tabular}{|c|c|c|}
\hline Model Parameters & $S[t]=0$ & $S[t]=1$ \\
\hline$P_{g g}$ & 0.9939 & 0.7202 \\
\hline$P_{g b}$ & 0.0061 & 0.2798 \\
\hline$P_{b g}$ & 1.000 & 0.6002 \\
\hline$P_{b b}$ & 0.000 & 0.3998 \\
\hline
\end{tabular}

\begin{tabular}{|c|c|c|}
\hline Comparison & Model & Exper. \\
\hline Errors per codeword & 27 & 25 \\
\hline Not decoded codewords & 16 & 16 \\
\hline
\end{tabular}

transition probabilities of the Markov model. The accuracy of the final burst error model is validated by comparing the model-generated numerical results with the experimental data. Table I shows the parameters of the Markov model, along with the results regarding the mean number of errors per codeword and the number of codewords that cannot be decoded in both cases. These results verify that the burst error model can be used to describe adequately the errors introduced due to the external disturbance.

2) Disturbance caused on a storage device when it is placed on a desk: The acceleration profile that affects the microscanner movement on both directions, as it is measured when a mobile phone that uses the storage device is placed on a desk, is presented in Fig. 8(a). Note that this profile is characterized by higher levels of acceleration and more steep value variations than the previous one. The deviation of the tip from the center of the line along with its effect on the readback signal in one data field is shown in Fig. 8(b). The locations of the errors that appear in one data field during the disturbance are shown in Fig. 8(c). Note that although the readback signal seems better than in the previous example, more errors occur. This is due to the fact that the deviation in both $\mathrm{x}$ and $\mathrm{y}$ axis reaches values of more than $40 \mathrm{~nm}$, which for the $\mathrm{x}$ direction means that different bits will be read leading to errors, irrespective of what the readback signal looks like. From the distribution of the bit errors, the External Impairment Level sequence that will be used to control the respective burst error model is derived. In this case, the definition of an extra level of impairment, $S[t]=2$, is needed to describe more accurately the errors distribution. The corresponding sequence is depicted in Fig. 8(c). Again, the errors from all data fields are used to calculate the transition probabilities of the Markov model. Table II shows the parameters of the Markov model, along with the mean number of errors per codeword and the number of codewords that cannot be decoded, as they are given by both the model and the simulator. In this case also, the results verify the accuracy of the burst error model.
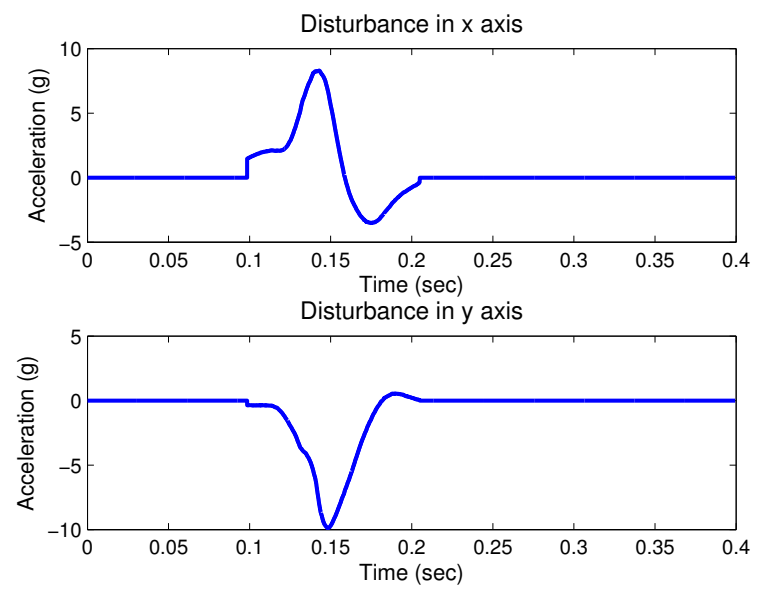

(a) External disturbance profile.
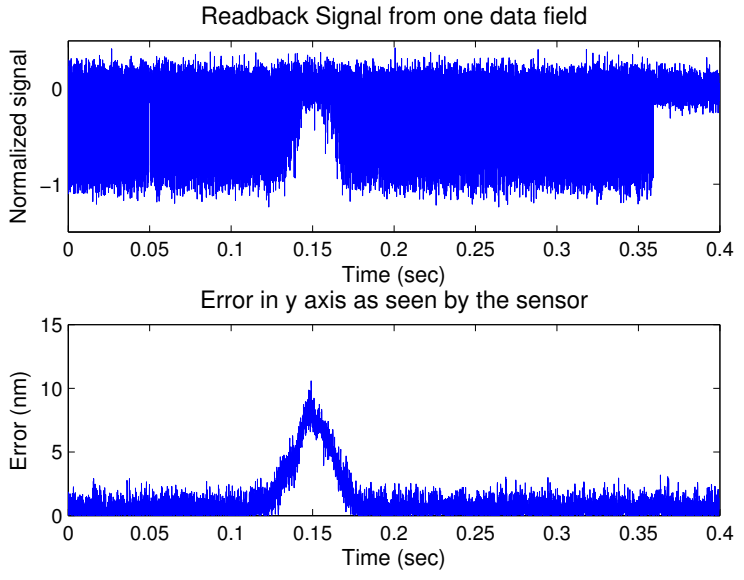

(b) Deviation from the center of the line in the y axis and its effect on the readback signal.
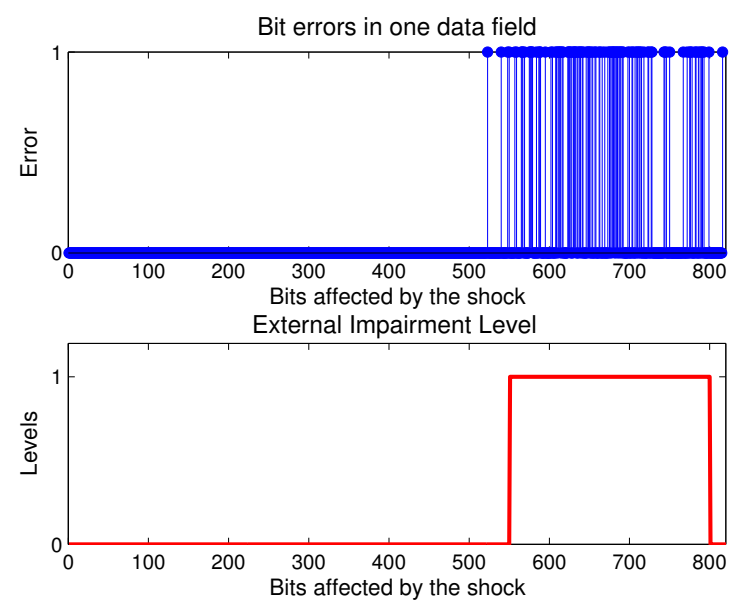

(c) Error bits observed in the shock duration and the corresponding External Impairment Level sequence.

Fig. 7. A probe-based storage device with $N=16$ data fields, 2048 bytes sector size, $M=16$ codewords and $R S(151,129)$ code is affected by an external disturbance measured experimentally when the device is attached on a person running with a speed of $10 \mathrm{~km} / \mathrm{h}$. 

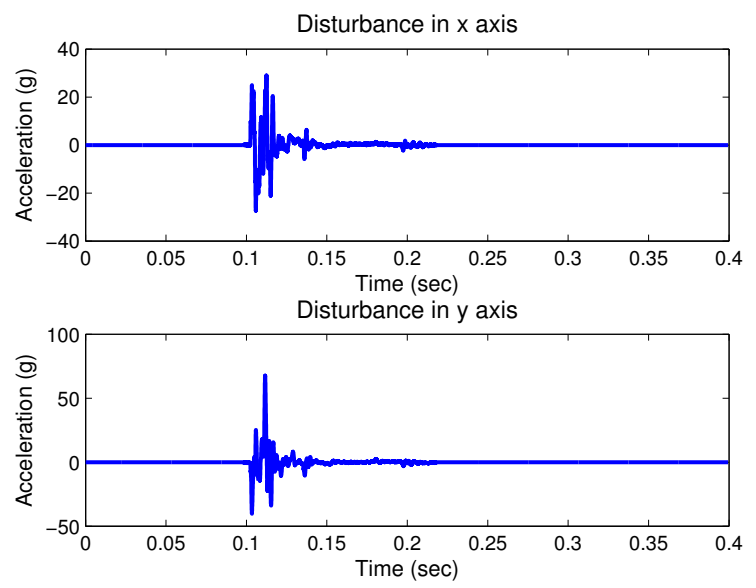

(a) External disturbance profile.
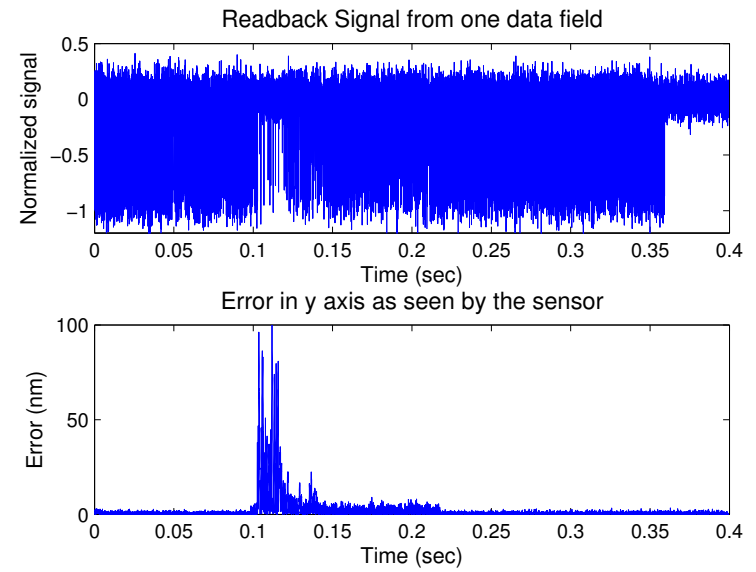

(b) Deviation from the center of the line in the $y$ axis and its effect on the readback signal.
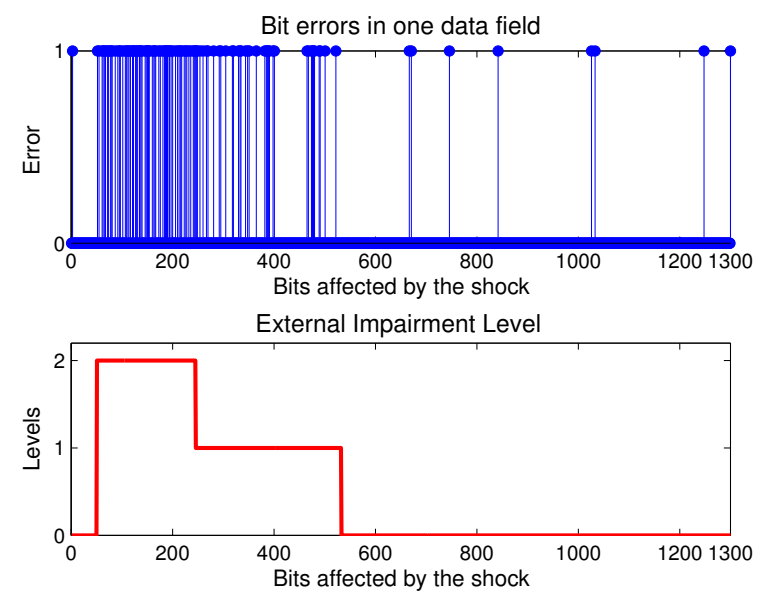

(c) Error bits observed in the shock duration and the corresponding External Impairment Level sequence.

Fig. 8. A probe-based storage device with $N=16$ data fields, 2048 bytes sector size, $M=16$ codewords and $R S(151,129)$ code is affected by an external disturbance measured experimentally when the device is placed on a desk.
TABLE II

MARKOV MOdel PARAMETERS AND COMPARATIVE RESUlTS WITH EXPERIMENTAL DATA FOR A SHOCK PROFILE CAUSED DURING PLACING THE DEVICE ON A DESK

\begin{tabular}{|c|c|c|c|}
\hline Model Parameters & $S[t]=0$ & $S[t]=1$ & $S[t]=2$ \\
\hline$P_{g g}$ & 0.9875 & 0.8364 & 0.5853 \\
\hline$P_{g b}$ & 0.0125 & 0.1636 & 0.4147 \\
\hline$P_{b g}$ & 1.000 & 0.9713 & 0.8936 \\
\hline$P_{b b}$ & 0.000 & 0.0287 & 0.1064 \\
\hline
\end{tabular}

\begin{tabular}{|c|c|c|}
\hline Comparison & Model & Exper. \\
\hline Errors per codeword & 47 & 48 \\
\hline Not decoded codewords & 16 & 16 \\
\hline
\end{tabular}

\section{CONClusions}

In this work, we presented a measurement-based burst error model for studying the effect of external disturbances on devices with multiple, simultaneously accessed storage fields. A set of Markov processes is used for modeling the burst errors that appear in the fields. For various kinds of disturbances, the correlation of the errors in the various channels and the parameters of the corresponding Markov processes are determined by measurements on an actual system. The application of the proposed model on a probe-based storage device with multiple storage fields was also presented and the accuracy of the model was proved for two different cases of external disturbances.

\section{REFERENCES}

[1] A. Pantazi, A.Sebastian, et al., "Probe-based ultrahigh-density storage technology," IBM J. Res. and Dev., vol. 52, no. 4/5, pp. 493-511, 2008.

[2] P. Vettiger, T. Albrecht, et al., "Thousands of Micro-Cantilevers for Highly Parallel and Ultra-Dense Data Storage," in Proc. IEDM 2003 IEEE Int'l Electron Devices Meeting 2003, Washington, DC, Dec. 2003, pp. pp. 32.1.1 - 32.1.4.

[3] M. Varsamou and T. Antonakopoulos, "A new data allocation method for parallel probe-based storage devices," IEEE Transactions on Magnetics, vol. 44, no. 4, pp. 547-554, Apr. 2008.

[4] E. Eleftheriou, T. Antonakopoulos, et al., "The Millipede, a MEMS-based scanning-probe data-storage system," IEEE Transactions on Magnetics, vol. 39, no. 2, pp. 938-945, Mar. 2003.

[5] G. Binnig, C. F. Quate, and C. Gerber, "Atomic force microscope," Phys. Rev. Lett, vol. 56, no. 9, pp. 930-933, 1986.

[6] H. Pozidis, W. Häberle, D. Wiesmann, U. Drechsler, M. Despont, T. Albrecht, and E. Eleftheriou, "Demonstration of thermomechanical recording at $641 \mathrm{Gbit} / \mathrm{in}^{2}$," IEEE Transactions on Magnetics, vol. 40, no. 4, pp. 2531-2536, Jul. 2004.

[7] P. Vettiger, G. Cross, et al., "The Millipede - Nanotechnology entering data storage," IEEE Transactions on Nanotechnology, vol. 1, no. 1, pp. 39-55, Mar. 2002.

[8] A.Sebastian, A. Pantazi, and H. Pozidis, "Jitter Investigation and Performance Evaluation of a Small-Scale Probe Storage Device Prototype," in Global Telecommunications Conference, 2007. GLOBECOM '07. IEEE, Washington, DC, USA, Nov. 2007, pp. 288-293.

[9] A.Sebastian, A. Pantazi, H. Pozidis, and E. Eleftheriou, "Nanopositioning for Probe-Based Storage Device," IEEE Control Systems Magazine, pp. 26-35, August 2008.

[10] A. Kotsopoulos and T. Antonakopoulos, "Modeling the Dynamics of MEMS-based Mobile Devices under Speed-controlled Human Motion Using Acceleration Measurements," in International Instrumentation and Measurement Technology Conference, 'I2MTC 2009', Singapore, May 2009. 